\title{
The Role of High Resolution Ultrasonography in Evaluation of Post Traumatic Peripheral Nerve Injury
}

\author{
Hesham M. Farouk $^{\text {a }}$, Alaa G. Youssef ${ }^{\mathrm{b}}$, Hamada M. Khater ${ }^{\text {a }}$
}

\begin{abstract}
${ }^{\mathrm{a}}$ Department of Radiology, Benha faculty of medicine, Benha University, Egypt. ${ }^{\mathrm{b}}$ Department of Radiology, New Cairo Hospital, New Cairo, Egypt.

Correspondence to: Alaa G. Youssef, Department of Radiology, New Cairo Hospital, New Cairo, Egypt.

Email:

elliejimy1990@gmail.com

Received: 8 February 2021

Background: With the development of scientific technology, applications of high-resolution ultrasound have rapidly developed in biomedical fields. High-resolution sonography clearly depicts peripheral nerve size spaces occupying lesions and anatomic variants along the entire length of the normative nerve. Aim: Assessment of efficacy of ultrasound in evaluation of degree of traumatic nerve injury. Methods This prospective study was carried out during the period from June 2019 to April 2020 and included a hundred participants. All patients were referred to the radiology department of Benha University from the emergency department and outpatient clinic of surgery department of Benha university hospital. Results: diagnosis and characterization of turmeric peripheral nerve injury using ultrasound Conclusion; US can be used to facilitate the preoperative diagnosis and planning of the optimal treatment strategy as it can permit tracing the injured nerve at the lesion site and the neuroma's extension. Finally, US has high accuracy for detection, diagnosis, assessment and treatment planning of traumatic peripheral nerve lesions.
\end{abstract}

Keywords: high resolution ultrasound, post traumatic peripheral nerve injury, nerve cut, neuroma, enlarged nerve.

\section{List of abbreviations; \\ US = Ultrasound \\ TPNI = traumatic Peripheral nerve injury}

\section{Introduction:}

The current approach for localizing and assessing the severity of traumatic peripheral nerve injuries involves clinical evaluation and electro diagnostic studies.
However, the ability of these approaches to determine the extent of nerve damage within the first 6 weeks after trauma is limited (1). Furthermore, the availability of 
Computed tomography (CT) and magnetic resonance imaging (MRI) neurography may be limited, and the costs can be significant. In comparison, ultrasonography is a portable, dynamic, and relatively low-cost technology (2).

With the development of scientific technology, applications of high-resolution ultrasound have rapidly developed in biomedical fields. High-resolution sonography clearly depicts peripheral nerve size spaces occupying lesions and anatomic variants along the entire length of the normative nerve. Using sonography, peripheral nerves have a fibrillar appearance, which is tape-like on longitudinal scans and ovoid on transverse scans(3).

Ultrasonographic patterns correlate well with histological structures. Normal peripheral nerves have a characteristic echotexture. Nerves are composed of hypoechoic fascicules within a hyperechoic environment (3).

High-resolution ultrasonography has been shown to support clinical and electrophysiological testing for detection of a variety of nerve abnormalities, including entrapment neuropathies, traumas, infectious disorders, and tumors (4). Since the earliest reports of peripheral nerve ultrasonography (5), progress in ultrasonography has been ongoing. Aside from neurophysiological assessment of peripheral nerve lesions, the technique of high-resolution ultrasonography provides an increasing amount of complementary morphological information about nerves and their surrounding tissues (6).

High-resolution ultrasonography images depict nerves in high image quality and enable clinicians to examine them along their anatomical course, thus giving valuable information about the origin and exact location of most lesions (7).

Additionally, ultrasonography provides the possibility of dynamic imaging of peripheral nerves. In the present study, we review the current state of high-resolution ultrasonography for imaging peripheral nerve traumatic peripheral nerve lesions with case illustrations (8).

Aim: Assessment of efficacy of ultrasound in evaluation of degree of traumatic nerve injury

\section{Patient and Methods}

This prospective study was carried out during the period from June 2019 to April 2020 and included a hundred participants. All patients were referred to the radiology department of Benha University from the emergency department and outpatient 
clinic of surgery department of Benha university hospital.

All patients included in the study were subjected to the following:

- Ethical committee approval was taken.

- Informed consent were obtained from all patients.

\section{Inclusion Criteria:}

Any patient presented with history of upper and lower limb and clinically suspected to have nerve injury.

\section{Exclusion criteria:}

Patients unwilling to complete the study.

* Patients not meeting the inclusion criteria.

Patients with associated other nerve lesions in forearm and hand other than trauma.

Patients with anything make examination impossible.

All participants were subjected to the following:

History taking.

Clinical provisional examination.

Neurological examination.

Electrophysiological studies.

Radiological investigation:

Ultrasound examination

Doppler examination

Surgical evaluation.

\section{Statistical analysis}

Analysis of data was done using SPSS version 20.0 (Chicago, Ill, USA). The data were summarized using descriptive statistics: mean, standard deviation, median, interquartile range for quantitative variables and number and percentage for qualitative values.

Also through Sensitivity $=$ true + ve/true + ve + false-ve $=$ ability of the test to detect positive cases.

Specificity $=$ true - ve $/$ true - ve + false + ve $=$ ability of the test to exclude negative cases.

\section{Results}

-Male represented $49 \%$ of the studied patients. Their age ranged from 25 to 45 years with mean 36.03 years

-Forty six percent of the studied patients had cutting trauma, $44 \%$ had non-cutting trauma and $10 \%$ had repeated trauma.

-Ninety percent of patients had nerves in upper limb affected.

- The most common nerve affected was median nerve (44\%) followed by ulnar nerve $(41 \%), 19 \%$ had radial nerve affected. Five percent had femoral and sciatic nerves affected and posterior tibial nerve was affected in 10\%.(Table 1) 
- Cross sectional area of affected nerves ranged from 14 to $28 \mathrm{~mm}^{2}$ with mean $19.323 \mathrm{~mm}^{2}$

- Forty three percent of patients had neuroma followed by swollen enlarged nerve $(44 \%)$.

- About three quarters of patients had no associated lesion .

- The Ultrasonography can predict eligibility for surgery in 45 out of The Ultrasonography can predict eligibility for surgery in 45 out of 47 patients who were truly eligible with $95.7 \%$ sensitivity and can rule out eligibility for surgery in all 4 patients with $100 \%$ specificity. Positive predictive value was $100 \%$ negative predictive value $66.7 \%$ and accuracy 96.1\%. Table (2)

\section{Case presentation}

Case number 1a cut in median nerve with nuroma incotenitly measured $10 \mathrm{~mm}$ with defect in sheath measured $2 \mathrm{~mm}$ Figure(1)

\section{Case number 2}

repeated non cutting truma by moderate tenosynovities in right hand lead to mild carpal tunel syndrome with cross sectional area of median nerve about $17 \mathrm{~mm} 2$ .Figure (2)

\section{Case number 3}

at site of injury nerouma measured $8 \times 2.5$ $\mathrm{mm}$ in one branch of meidian nerve.

Figure(3)

\section{Case number 4}

At site of amputation multiples nerouma detected at femoral and sciatic nerve Largest sciatic measured $46 \mathrm{~mm}$ and terminal femoral one measured $15 \mathrm{~mm}$ .Figure (4)

Table (1): Distribution of the studied patients according to nerve:

\begin{tabular}{lll}
\hline Nerve & N & \% \\
\hline Ulnar nerve & 41 & 41 \\
Radial nerve & 19 & 19 \\
Median Nerve & 44 & 44 \\
Digital Nerve & 5 & 5 \\
Posterior Tibial Nerve & 10 & 10 \\
Femoral \& Sciatic Nerves & 5 & 5 \\
\hline
\end{tabular}


Table (2): Validity of US in diagnosis of eligibility for surgery among the studied patients:

\begin{tabular}{cccc}
\hline \multirow{2}{*}{ US } & \multicolumn{2}{c}{ Surgery } & Total \\
\hline Positive & Positive & Negative & 45 \\
Negative & 45 & 0 & 6 \\
Total & 2 & 4 & 51 \\
& 47 & 4 & Value \\
& & & $95.7 \%$ \\
& Sensitivity & & $100 \%$ \\
& Specificity & & $100 \%$ \\
& PPV & & $66.7 \%$ \\
& NPV & & $96.1 \%$
\end{tabular}

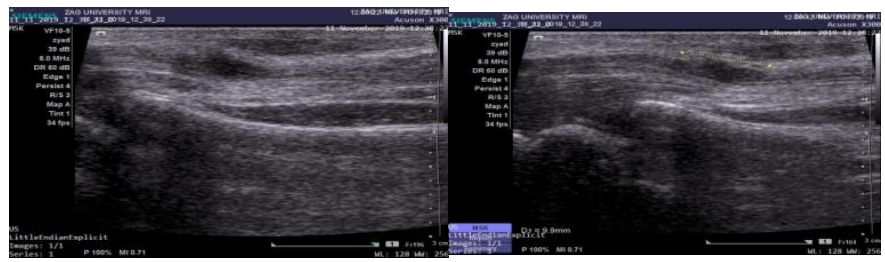

figure (1) a cut in median nerve with nuroma incotenitly measured $10 \mathrm{~mm}$ with defect in sheath measured $2 \mathrm{~mm}$

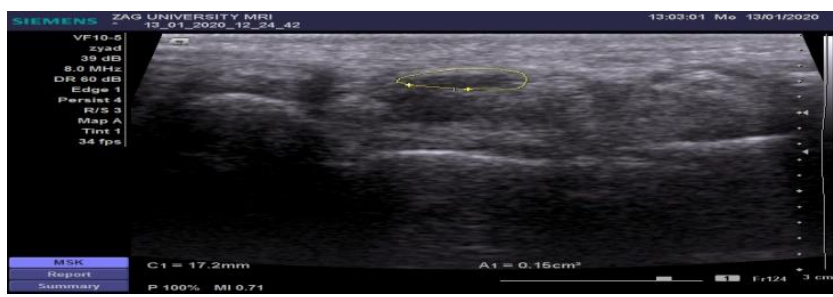

Figure (2) mild carpal tunel syndrome with cross sectional area of median nerve about $17 \mathrm{~mm} 2$

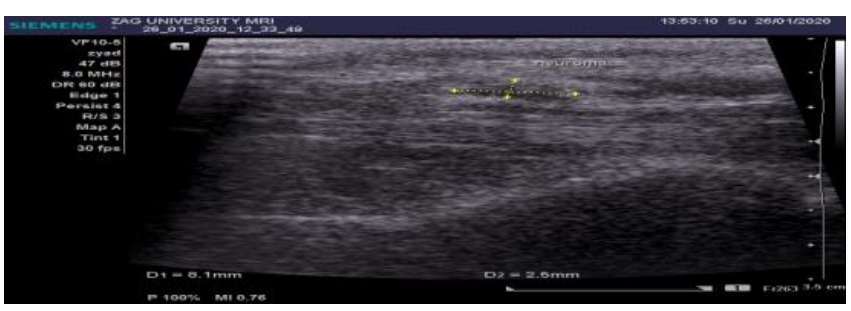

Figure (3) nerouma measured $8 \times 2.5 \mathrm{~mm}$ in one branch of meidian nerve. 


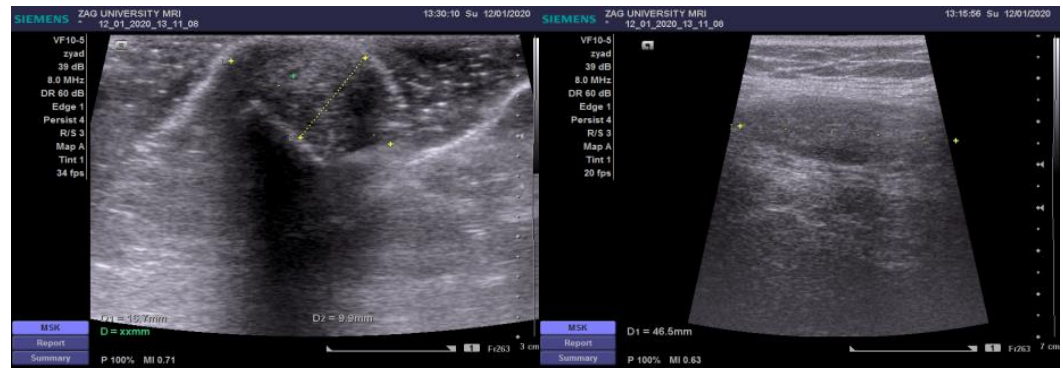

Figure (4) nerouma detected at femoral and sciatic nerve Largest sciatic measured $46 \mathrm{~mm}$ and terminal femoral one measured $15 \mathrm{~mm}$

\section{Discussion}

Traumtic injuries of peripheral nerves are increasingly recognized in today's clinical practice, because of improved trauma services (9).

Traumatic Peripheral nervous system injuries (TPNI) can result in multiple pathologies, with involvement of single or multiple nerve units and subsequent sensory and/or motor impairment (10)

TPNI in the upper limb significantly impact individuals' function and ability to return to work (11).

An understanding of the classification, pathophysiology, and electrodiagnosis of these lesions is critical to the appropriate diagnosis, localization, and management of peripheral nerve trauma (12).

US is now considered an optimal imaging technique to evaluate the normal anatomy and disorders of peripheral nerves (13).
Recent rapid technical advances in US, such as new ultra-high frequency probes and smaller probe sizes, have led to improved image quality. This, in turn, has accelerated the growth of musculoskeletal US. Known advantages of US are its lack of ionizing radiation, noninvasiveness, portability and low cost (14).

The improved resolution and wide availability of ultrasound (US) instruments have made this tool useful in assessing nerve diseases and this may be particularly true in traumatic lesions (15).

Ultrasonography not only allows one to precisely localize the site of nerve injury. it also indicates whether a nerve is completely transected or partially dissected or 
Whether the nerve is displaced or even encased by surrounding scar formation or by a fibrous (16).

So, accurate localization and the assessment of severity of the lesion by Sonography played an important role in the decision making, whether to surgically inspect the nerve or not (17).

The aim of this work was to describe the role of high frequency US in diagnosis and assessment of traumatic median, ulnar, radial, digital, sciatic and femoral nerve injuries .

In our study, we included 100 patients who sustained trauma to the forearm and the hand and lower limb clinically suspected to have peripheral nerves injury. Their age range from 25 to 45 years old, and mean age was $36.3 \pm 5.625$ years. Out of the 100 patients included, 51 were males $(51 \%)$, and 49 were females $(49 \%)$ with male to female ratio (1.01:1).

In accordance with our study a study done on 2009 concluded that the peripheral nerve injuries were seen predominantly in young men and in the most productive age group. Trauma-related PNIs were clearly less common after the age of seventy.

The most common clinical presentation in patients was tingling and numbness which is noted in 75 cases $(75 \%)$.
In another study it was stated that stated that Ultrasonography was able to correctly identify the site of nerve trauma in 6 patients presented with peripheral nerve (18).

Trauma stretch injury, nerve compression from trauma, or iatrogenic injury from previous surgical exploration; in all cases, ultrasonography demonstrated correct lesion diagnosis and location (100\%).

In agreement with previous study, we correctly detected the location of nerve injury by US evaluation. Nerve injury at the upper limb was the most common affected site; detected in 90 cases (90\%) median nerve was the most affected 44 cases (44\%) digital nerve was the least affected in upper limb 5 cases (5\%), nerves of lower limb less affected then upper limb the most affected posterior tibial nerve 10 cases $(10 \%)$ and the least affected sciatic nerve 5 cases (5\%).

In the study which included 104 patients that underwent surgery after forearm nerve injury and the majority were males, injury by a sharp object was the most frequent etiologic factor and it occurred in (59.6\%) of patients (19). Concerning associated injuries, patients had muscle or tendon injury of the forearm, but forearm bone fracture and vascular trauma were also frequent. The total number of patients 
with associated injuries was $45.2 \%$ of the total number of patients.

In similar result in our study, nerve injury by sharp objects and lacerations mostly RTA were the most common types of nerve injury, sharp object was encountered in 46 injured nerves $(46 \%)$, 43 of them formed neuroma (43\%), 3 of them complete cut of nerve (3\%)

Nerve injury was associated with tendon, bony or vascular injuries and synovitis and other pathology 25 injured nerves (25\%) while 25 injured nerves $(67.7 \%)$ were not.

It was mentioned that high resolution and color doppler was used to evaluate 6 patients suffered from trauma of the upper limb nerves. The Most frequently injured nerve was median nerve, followed by ulnar nerve, then radial nerve. The most common sonographic features observed in nerve trauma was nerve thickening. The epineurium was seen as an echogenic line surrounding the nerve. Other sonographic features seen were the development of a scar tissue, partial and complete discontinuity in the epineurium, \& side and end neuroma. The neuroma appeared as a hypoechoic structure with no vascularity (20).
According to some studies (21 \& 22), combined lesion most commonly involved the ulnar and median nerves.

This findings was significantly proved in this study where Median nerve was the most common injured nerve in our patients detected in 44 (44\%), followed by the ulnar nerve injury in 41 patients (41\%) while radial nerve injury was found in 19 patients (19\%) and combined median and ulnar nerve injuries was found in 10 patient $(10 \%)$.

In a study done some years ago, it was concluded that High-resolution ultrasonography, has been used successfully to evaluate 14 cases of traumatic peripheral nerve injury preoperatively. It revealed axonal swelling, neuroma formation, complete nerve transaction prior to surgery and improvement of subsequent surgical planning. These findings were $100 \%$ in agreement with intra operative findings (23).

As for, preoperative and intraoperative ultrasonography for upper extremity peripheral nerve lesions used in the description of the degree of injury, determination of complete or incomplete nerve sectioning, the presence of foreign body, the continuity of the nerve, 
determination of nerve stumps and scar tissue, and the presence of neuroma (23).

In a study on 98 patients with trauamtic nerve lesions, US specifically contributed to the (1) assessment of nerve continuity/discontinuity (2) identification of aetiology; and (3) demonstration of multiple sites of damage. US allows evaluating the true distance between the useful nerve ends (excluding neuromas or frayed nerve endings that surgically must be excluded).the study showed that ultrasound was able to aid in the diagnosis or modification of the therapeutic path in $60 \%$ of patients with traumatic nerve lesions and was contributive mainly in cases with neurophysiological evidence of complete axonal damage. Anatomical information is often crucial for choosing the most appropriate therapeutic strategies (and for surgical planning) (24)

In 2015, it was concluded that US can provide the following: 1) whether the nerve remains in continuity; 2) length of the gap, if present; 3) presence of a focal neuroma and its location/size; 4) additional areas of nerve injury, such as tandem lesions; 5) degree of adjoining scar tissue; and 6) status of adjoining tissues such as blood vessels and bone (25).
In another study it was stated that high resolution ultrasongraphy can be helpful in evaluation of 26 patients with peripheral nerve lesions of the upper limb. Ultrasonographic examination demonstrated loss of continuity of a nerve bundle, the formation of a neuroma of a stump, and partial laceration of a nerve with loss of the normal fascicular pattern. There was agreement between US finding and intraoperative findings in all cases (26).

In agreement with previous studies; we found that The majority of cases (44\%) of cases showed disruption of fascicles with intact epineurium with thickening of nerve axons, (3\%) of cases had loss of continuity in complete transection, (43\%) of cases had terminal neuroma, (5\%) of cases showed loss of fascicles but had no loss of continuity..

In our study, neuroma incontinuity was the most prevalent finding by US.it was found in 43 injured nerves $(43 \%)$. Followed by swollen enlarged nerve (thickening ) after repeated trauma in 39 injured nerves (39\%).proximal neuroma was found in 5 injured nerves $(5 \%)$.Foreign bodies were found in 3 injured nerves (3\%) and 2 injured nerve. Entrapment by bony fragment and incomplete tear are the least common 
findings found only in two injured nerve $(2 \%)$.

Foreign bodies appeared as hyperechoic structures with acoustic shadowing; a comet tail artifact can be seen if metallic fragments exist. The reflectivity of a foreign body depends on its acoustic impedance on US. Therefore, metal and glass are significantly more reflective than glass and wood, respectively. As a result of reactive inflammation or granuloma formation, a hypoechoic area surrounding the foreign body can be demonstrated in subacute and chronic lesions.

It was stated that US is playing an increasingly important role in the diagnostic process for detection and localization of foreign bodies, All soft tissue foreign bodies (glass, metal, stone, plastic, wood) are hyperechoic on US, with a surrounding hypoechoic area corresponding to granulation tissue, edema, or hemorrhage. Posterior acoustic shadowing or comet-tail artifact may be present depending on the type of material (27)

This agree with our study as we found 3 cases with foreign body impaction is above the wrist joint and the other two are at the upper third of forearm . FB mostly appeared on US as localized hyperechoic foci with posterior acoustic shadowing or comet tail sign or surrounded by hypoechoic halo due to granulation.

According to the study done on 2014 (10) , high resolution ultrasound demonstrated high sensitivity and specificity for the detection of specific pathologies of peripheral nerve injuries and its ability to differentiate between them.

That was matched with the results of our study, high frequency ultrasound had high sensitivity $95.7 \%$ and specificity $100 \%$ and overall accuracy $96.1 \%$ in diagnosis of traumatic nerve injuries. this means that preoperative US had $96.1 \%$ accuracy for detection of same structural changes found during operation in cases of traumatic nerve injury.

In the study done on 24 patients with 29 traumatic nerve injuries using new high frequency ultrasound. Overall, the ultrasound and clinical results were in agreement for 28 of the 29 nerves (96\%). Limitations were because of extensive fibrosis (28).

In a prospective study of 202 patients with a history of trauma; all patients underwent US and electrodiagnosis, 117 cases that underwent operative treatment, the accuracy of classification by using US was $93.2 \%$. There were 8 cases 
misclassified by US, in which minimal scar tissue was seen and surgery revealed nerve degeneration (29). In our study, preoperative US findings had agreed with intra operative findings in 47 injured nerves from 51 intra operative .

We had 4 cases $(8 \%)$ ut of the 51 cases with misdiagnosis, one case with traumatic neuropathy and swollen nerve was diagnosed as neuroma secondary to partial nerve injury on US. One case on US, in which partial injury was seen but where surgery revealed a complete tear.

One of the limitations of ultrasonographic nerve assessment is that the peripheral nerves cannot be traced clearly throughout their entire paths and require an experienced radiologist with a thorough knowledge of the ultrasonographic appearance of different soft-tissue structures.

Overall Based on our findings, we believe that high-frequency ultrasonography provides an economical and accurate imaging modality utilized in the diagnosis, assessment and treatment planning of traumatic peripheral nerve lesions.

\section{Conclusion}

Overall, our study concluded that high frequency ultrasound had high sensitivity $995.7 \%$ and specificity $100 \%$ and overall accuracy $96.1 \%$ in diagnosis of traumatic nerve injuries.

Ultrasonography not only allows one to precisely localize the site of nerve injury, it also indicates whether a nerve is completely transected or partially dissected or whether the nerve is displaced or even encased by surrounding scar formation or by a fibrous or bony callus after bone fracture. Furthermore, ultrasonography may identify fracture fragments compressing nerves in close vicinity to bone fractures, detect foreign bodies or may quantify the amount of nerve retraction after complete nerve transection.

US can be used to facilitate the preoperative diagnosis and planning of the optimal treatment strategy as it can permit tracing the injured nerve at the lesion site and the neuroma's extension.

Finally, US have high accuracy for detection, diagnosis, assessment and treatment planning of traumatic peripheral nerve lesions. 


\section{References}

1. Cartwright MS, Shin HW, Passmore LV, Walker FO. (2007):Ultrasonographic findings of the normal ulnar nerve in adults. Arch Phys Med Rehab. 2007a;88:394-396.

2. Lee FC, Singh H, Nazarian LN, JK Ratliff. (2011): High-resolution ultrasonography in the diagnosis and intraoperative management of peripheral nerve lesions: clinical article. Journal of neurosurgery, 114(1): 206-211

3. Kleinert JM, Mehta S (1996) Radial nerve entrapment. Orthop Clin North Am 27:305-315

4. Bacigalupo L, Bianchi S, Valle $M$, Martinoli C (2003): Ultrasonography of peripheral nerves. Radiologe 43:841-849.

5. Solbiati L, De Pra L, Ierace T, Bellotti E, Denchi LE(1985): Highresolution sonography of the recurrent laryngeal nerve: anatomic and pathologic considerations. AJR Am J Roentgenol 145:989-993

6. Fornage BD(1988): Peripheral nerves of the extremities: imagin with US. Radiology 167:179-182

7. Chiou HJ, Chou YH, Chiou SY, Liu JB, Chang CY(2003): Peripheral nerve lesions: role of high-resolution US. Radiographics 23:E15, 2003

8. Martinoli C, Bianchi S, Cohen M, Graif M(2005): Ultrasound of peripheral nerves. J Radiol 86:1869-1878.

9. Peer $S$ and Bordner $G$ (editors) (2008): High resolution sonography of the peripheral nervous system, $2^{\text {nd }}$ ed.Berlin: SpringerVerlag.

10.Alaqeel A and Alshomer F (2014): High resolution ultrasound in the evaluation and management of traumatic peripheral nerve injuries: review of the literature. Oman Medical Journal, 29)5):314-319

11.Miller T and Reinus W (2010): Nerve entrapement syndromes of the elbow, forearm and wrist, AJR Am J Roentgenol 195(3): 58594.

12. Robinson LR (2000): Traumatic injury to peripheral nerves. Muscle \& nerve, 23(6): 863-873

13. Beekman R and Visser LH (2004): "Highresolution sonography of the peripheral nervous system-a review of the literature." European Journal of Neurology 11(5):305-314.

14.Wong DC, Wansaicheong GK and Tsou IY. (2009): Ultrasonography of the hand and wrist. Singapore Med J, 50(2):219-225.

15. Martinoli C, Bianchi S, Prato N, (2008): Ultrasonography of Peripheral Nerves Seminars in Ultrasound, CT and MR; Vol.121, No.3, pp.205-213.

16. Schminke U (2012): Ultrasonography of peripheral nerves-Clinical significance. Perspectives in Medicine, 1(1):422-426.

17.Eser F, Aktekin LA, Bodur H ,C Atan. (2009): Etiological factors of traumatic peripheral nerve injuries. Neurology India, 57(4):434-437.

18. Lee FC, Singh H, Nazarian LN ,JK Ratliff. (2011): High-resolution ultrasonography in the diagnosis and intraoperative management of peripheral nerve lesions: clinical article. Journal of neurosurgery, 114(1): 206-211.

19. Rasulić L, Puzović V, Rotim K, M Jovanović , M Samardžić, Bojana Živković, et al. (2015): The epidemiology of forearm nerve injuries--a retrospective study. Acta clinica Croatica, 54(1):19-24.

20.Afsal M, Chowdhury V, Prakash A,Sapna Singh , Neera chowdhury. (2016): Evaluation 
of peripheral nerve lesions with highresolution ultrasonography and color Doppler. Neurology India, 64(5):1002-9001.

21.Campbell WW (2008): "Evaluation and management of peripheral nerve injury." Clinical neurophysiology 119(9): 1951-1965

22.Kouyoumdjian JA (2006): Peripheral nerve injuries: a retrospective survey of 456 cases. Muscle \& nerve, 34(6), 785-788.

23. Cokluk C and Aydin K (2007): Ultrasound examination in the surgical treatment for upper extremity peripheral nerve injuries: part I. Turk Neurosurg Oct; 17(4):277-282.

24.Padua L, Di Pasquale A, Liotta G, G. Granata ,C.Pazzaglia, D.Coraci, et al. (2013): Ultrasound as a useful tool in the diagnosis and management of traumatic nerve lesions. Clinical Neurophysiology, 124(6):1237-1243.

25.Zeidenberg J, Burks SS, Jose J,Ty K.Subhawong , Allan D.Levi. (2015): "The utility of ultrasound in the assessment of traumatic peripheral nerve lesions: report of 4 cases." Neurosurgical focus 39(3):E3.

26. Toros T, Karabay N, Ozaksar K,T.S.Sugun ,M.Kayalar, E.Bal. (2009): evaluation of the peripheral nerve injuries in upper limb with ultrasonography; Bone \& Joint Journal, 91(6):762-765.

27.Dumarey A, De Maeseneer $M$ and Ernst C (2004): Large wooden foreign body in the hand: recognition of occult fragments with ultrasound. Emergency radiology, 10(6):337-339.

28.Hollister AM, Simoncini A, Sciuk A,Jenee Jordan. (2012): High frequency ultrasound evaluation of traumatic peripheral nerve injuries. Neurological research, 34(1):98103.

29.Zhu J, Liu F, Li D, J Shao, B Hu. (2011): Preliminary study of the types of traumatic peripheral nerve injuries by ultrasound. European radiology, 21(5):10971101.

To cite this article: Hesham M. Farouk, Alaa G. Youssef, Hamada M. Khater. The Role of High Resolution Ultrasonography in Evaluation of Post Traumatic Peripheral Nerve Injury. BMFJ 2021; 38(2): 699-711. DOI: 10.21608/bmfj.2021.62118.1382 
Benha medical journal, vol. 38, issue 2, 2021 